Short Communication

\title{
Inhibition of VEGF mediated post receptor signalling pathways by recently developed tyrosine kinase inhibitor in comparison with sunitinib
}

\author{
Roman Moravčík ${ }^{1}$, Katarína Stebelová ${ }^{1}$, Andrej Boháč̌,3 and Michal Zeman ${ }^{1}$ \\ ${ }^{1}$ Department of Animal Physiology and Ethology, Faculty of Natural Sciences, Comenius University in Bratislava, \\ Mlynská dolina, Ilkovičova 6, 84215 Bratislava, Slovak Republic \\ ${ }^{2}$ Department of Organic Chemistry, Faculty of Natural Sciences, Comenius University in Bratislava, Mlynská dolina, \\ Ilkovičova 6, 84215 Bratislava, Slovak Republic \\ ${ }^{3}$ Biomagi, Ltd., Mamateyova 26, 85104 Bratislava, Slovak Republic
}

\begin{abstract}
Inhibition of angiogenesis involves blocking of tyrosine kinases (TK) implicated in signalling of vascular endothelial growth factor receptors (VEFGR). The inhibition of TK results in a disruption of Ras/Raf/MEK/ERK1/2 and PI3K/Akt signalling pathways. We evaluated recently developed TK inhibitor 22SYM and compared its anti-angiogenic effects with an approved multitargeted TK inhibitor sunitinib L-malate (sunitinib). Both compounds significantly inhibited migration and proliferation of human umbilical vein endothelial cells and ERK1/2 and Akt phosphorylation induced by VEGF. The lower inhibitory activity of 22SYM probably reflects its lower bioavailability and higher specific binding to VEGFR2 TK, which may decrease its potential side effects and toxicity in comparison with sunitinib.
\end{abstract}

Key words: Tumour angiogenesis - ERK1/2 - Akt - Sunitinib - HUVEC

Angiogenesis, the formation of new blood vessels from preexisting vasculature is a complex process, which includes endothelial cell proliferation, migration, differentiation and basement membrane degradation (Carmeliet and Jain 2000). Under physiological conditions it has an essential role in development, reproduction and tissue repair. By contrast, pathological angiogenesis is involved in tumour growth and metastases (Folkman 2007).

The major regulators of both physiological and pathological angiogenesis are vascular endothelial growth factors (VEGFs), among which VEGF-A plays the most important role (Ferrara 2010). VEGFs can act via several tyrosine kinase (TK) receptors among which VEGFR2 is essential for endothelial cell biology. VEGFs are mitogenic for endothelial cells and stimulates their proliferation and migration through VEGFR2-induced activation of the Ras/Raf/

Correspondence to: Roman Moravčík, Department of Animal Physiology and Ethology, Faculty of Natural Sciences, Comenius University in Bratislava, Mlynská dolina, Ilkovičova 6, 84215 Bratislava, Slovak Republic

E-mail: moravcikr@fns.uniba.sk
MEK/ERK1/2 pathway (Zachary 2001). Another important pathway responsible for cell survival is the phosphatidylinositol 3-kinase (PI3K)/Akt signal transduction pathway since Akt phosphorylates Bcl-2 associated death promoter and caspase 9 and thereby inhibits their apoptotic activity (Cantley 2002).

Activation of VEGFR2 by VEGF-A modulates signalling pathways and biological processes, which have a crucial role in tumour angiogenesis and therefore VEGF-A/VEGFR2 are the main targets to diminish tumour neovascularisation. Anti-angiogenic therapy has been developed with the aim to act against tumour activated endothelial cells instead of cancer cells (Faivre et al. 2007). Recently, two approaches have been used to inhibit angiogenesis. The first approach involves inhibition of pro-angiogenic growth factors with monoclonal antibody and the second approach involves multitargeted TK inhibitors, such as sunitinib L-malate (Sutent $^{\oplus}, 2006$ Pfizer; later mentioned as sunitinib), which binds to VEGFR TK and other TK (Faivre et al. 2007). The advantage of multitargeted anti-angiogenic therapy consists in a disruption of several independent biological pathways, which are vital for tumour proliferation and metastasis (Ivy 
et al. 2009). Although a number of anti-angiogenic inhibitors is available, currently used compounds failed to meet original expectations and there is still a need to develop new and more effective small TK inhibitors.

The aim of the present study was to evaluate mechanisms of anti-angiogenic activity of newly developed TK inhibitor 22SYM and compare its efficiency with the approved multitargeted tyrosine kinase inhibitor sunitinib.

Human umbilical vein endothelial cells (HUVECs) were isolated from a fresh umbilical cord and cultured in endothelial cell growth medium (ECGM) with growth supplements (PromoCell, Germany). Cultures were maintained in a 5\% $\mathrm{CO}_{2}$ humidified incubator at $37^{\circ} \mathrm{C}$.

Migration and proliferation of cultured HUVECs were determined by wound healing assay. The cells were cultured in 24-well plates (TPP, Switzerland) coated with $1.5 \%$ gelatine (Sigma-Aldrich, USA). After reaching $80 \%$ of confluence, the culture medium was replaced with the starvation medium (M199 containing 2\% fetal bovine serum, $5 \mathrm{UI} / \mathrm{ml}$ of heparin and antibiotics). After $17 \mathrm{~h}$, monolayer of each well was wounded and cells were incubated in the starva- tion medium containing $40 \mathrm{ng} / \mathrm{ml} \mathrm{VEGF}_{165}$ (Sigma Aldrich, USA) and indicated concentrations of sunitinib or 22SYM. The recovered area was observed with an inverted optical microscope Olympus IMT2 (Olympus, Japan) and recorded by a camera system Moticam 1000 (Motic Incorporation, Hong Kong) at zero time and after $9 \mathrm{~h}$ of incubation. Photographs were evaluated by software Motic Images Plus 2.0 (Motic Incorporation, Hong Kong).

In further experiments HUVECs at passage 1-2 were used. They were cultured in 6-well plates to reach confluence. Subsequently ECGM was replaced with the starvation medium for $17 \mathrm{~h}$ and cultured in the starvation medium containing indicated concentrations of sunitinib or 22SYM for 1 hour. Afterwards, the cells were treated with $40 \mathrm{ng} / \mathrm{ml}$ of $\mathrm{VEGF}_{165}$ for $10 \mathrm{~min}$ and lysed in a lysis buffer. Proteins were separated in SDS-PAGE and transferred to a polyvinylidene fluoride (PVDF) membrane (Pall Incorporation, Austria). Blots were probed with anti-phospho-p44/42 MAPK (ERK1/2), anti-p44/42 MAPK (ERK1/2), antiphospho-Akt, anti-Akt and peroxidase-labelled anti-rabbit and anti-mouse secondary antibodies. All antibodies were

$\mathbf{A}$

sunitinib $10 \mu \mathrm{M}$

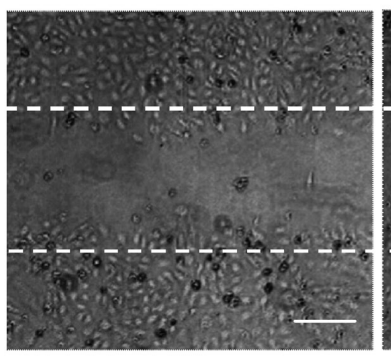

22SYM $100 \mu \mathrm{M}$

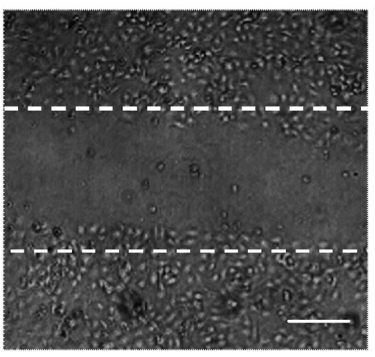

22SYM $10 \mu \mathrm{M}$

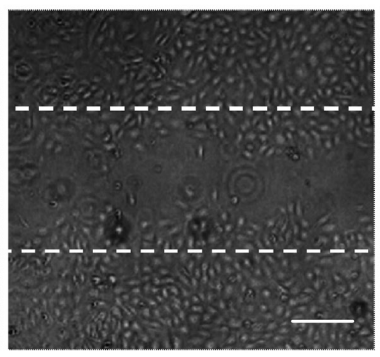

VEGF

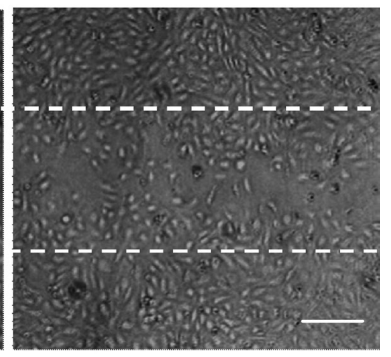

B

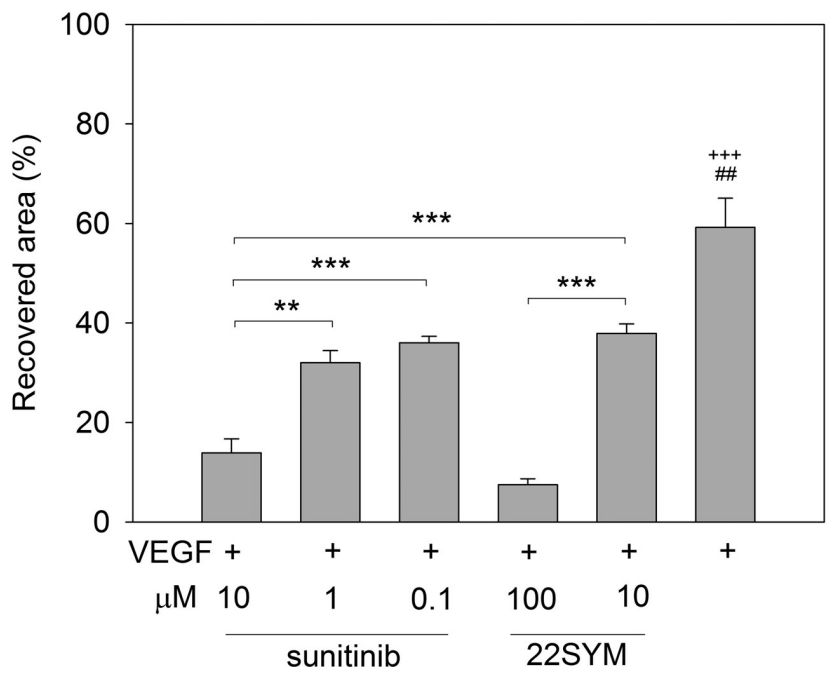

Figure 1. The intensity of HUVECs migration and proliferation $9 \mathrm{~h}$ after treatment with sunitinib and 22SYM. A. Confluent HUVECs monolayers were wounded $17 \mathrm{~h}$ after starvation in a fresh starvation medium with indicated concentrations of sunitinib and 22SYM in the presence of $40 \mathrm{ng} / \mathrm{ml} \mathrm{VEGF}{ }_{165}$. Photographs were taken at the beginning and after $9 \mathrm{~h}$ of incubation. White dashed lines indicate the edge of wounds. The line situated on the right down in each picture indicate the wound edge scale $(\mathrm{bar}=300 \mu \mathrm{m})$. B. The regrowth of HUVECs into the cell-free area was measured after $9 \mathrm{~h}$. Percentage of recovered area are expressed as mean \pm SEM for $n=8$. ${ }^{* *} p<0.01,{ }^{* *} p<0.001$ for indicated groups; $+++p<$ 0.001 VEGF stimulated (control) group compared to $10 \mu \mathrm{M}$ sunitinib, $1 \mu \mathrm{M}$ sunitinib and $100 \mu \mathrm{M} 22 \mathrm{SYM}$; \#\# $p<0.01$ VEGF stimulated (control) group compared to $0.1 \mu \mathrm{M}$ sunitinib and $10 \mu \mathrm{M} 22 \mathrm{SYM}$. 
obtained from Cell Signaling Technology (USA). Proteins were detected using enhanced chemiluminescence reagent ECL Prime $^{\mathrm{TM}}$ (Amersham Biosciences, UK) and developed on Amersham Hyperfilm (Amersham Biosciences, UK). The ratio between density of total and phosphorylated forms of proteins was determined using Quantity One software (Bio-Rad, USA).

Data were analysed statistically with one way ANOVA followed by a post hoc test $(p<0.05$ considered as significant).

In the wound healing assay $\mathrm{VEGF}_{165}$ significantly stimulated recovery of wounded area after $9 \mathrm{~h}$ (Fig. 1). Sunitinib as well as 22SYM inhibited migration and proliferation of HUVECs in a dose dependent manner. Similar wound recovering areas (61 $\pm 2 \%$ and $64 \pm 3 \%)$ were observed after treatment with sunitinib at $0.1 \mu \mathrm{M}$ and $22 \mathrm{SYM}$ at $10 \mu \mathrm{M}$ concentrations suggesting that 22SYM is a weaker inhibitor than sunitinib in the wound healing assay.

A

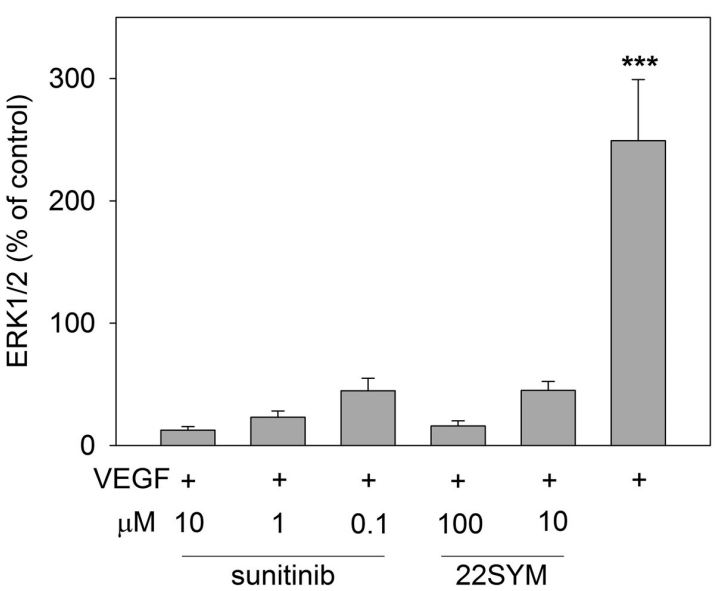

B

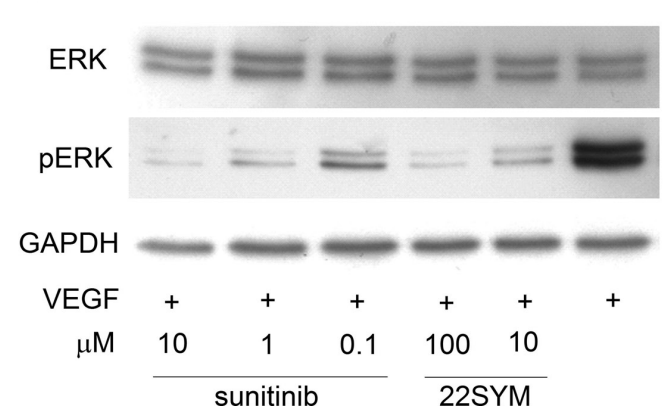

Both compounds suppressed VEGF-induced phosphorylation of Akt in a dose-dependent manner. Suppression of ERK1/2 by both compounds was greater than that of Akt (Fig. 2). Sunitinib in concentration $10 \mu \mathrm{M}$ inhibited ERK1/2 phosphorylation at $95 \%$ and Akt at $85 \%$ as compared with the VEGF stimulated control. 22SYM in concentration $10 \mu \mathrm{M}$ inhibited phosphorylation of ERK1/2 at $82 \%$ and phosphorylation of Akt at $45 \%$ in comparison with the VEGF stimulated control (Fig. 2A).

Blockade of angiogenesis is an important therapeutic strategy for cancer treatment. In this process, inhibition of endothelial cell proliferation, migration, survival and tumour vessel maturation is crucial (Carmeliet and Jain 2000). It is also well known that multikinase inhibitors like sunitinib inhibits ERK1/2 and Akt signalling pathways in cancer cell lines and has direct antitumour activity (Faivre et al. 2007). In this study we demonstrate that 22SYM inhibits VEGFinduced signalling pathways responsible for cell proliferation, migration and survival in HUVECs.
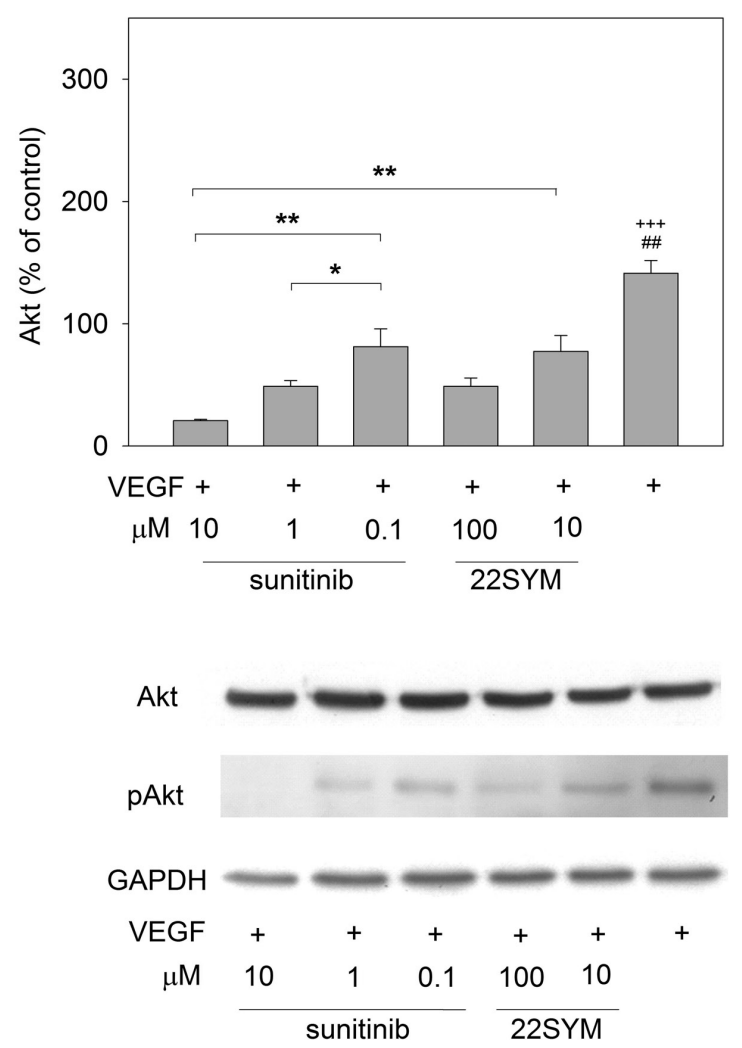

Figure 2. Inhibition of ERK1/2 and Akt phosphorylation after administration of indicated concentrations of sunitinib and 22SYM in the presence of $40 \mathrm{ng} / \mathrm{ml} \mathrm{VEGF}_{165}$. A. The ratio between density of total and phosphorylated forms of ERK1/2 and Akt expressed as the mean percentage of VEGF-stimulated group (control) \pm SEM for $n=3 .{ }^{*} p<0.05,{ }^{* *} p<0.01,{ }^{* *} p<0.001$ for indicated groups; ${ }^{+++} p<0.001$ VEGF stimulated (control) group compared to $10 \mu \mathrm{M}$ sunitinib, $1 \mu \mathrm{M}$ sunitinib and $100 \mu \mathrm{M} 22 \mathrm{SYM}$; ${ }^{\# \#} p<0.01 \mathrm{VEGF}$ stimulated (control) group compared to $0.1 \mu \mathrm{M}$ sunitinib and $10 \mu \mathrm{M} 22 \mathrm{SYM}$. B. Representative Western blot illustrating density of total and phosphorylated forms of ERK1/2 and Akt after administration of sunitinib and 22SYM. 
Our observations showed a dose-dependent decrease of phosphorylation of Akt and ERK1/2 kinases in HUVECs. For the first time we compared properties of new compound 22SYM with clinically approved anti-angiogenic inhibitor sunitinib.

Our results showed that 22SYM as well as sunitinib inhibit both ERK1/2 and Akt phosphorylation. However, both inhibitors did not affect both cascades with the same power. The novel angiogenesis inhibitor 22SYM acts predominantly by inhibition of VEGFR2-mediated pathways (Lintnerová et al. 2014) but post-receptor steps have not been characterized yet. Our results demonstrated that the inhibition of phosphorylation of both kinases Akt and ERK1/2 is involved. Sunitinib modulates an activity of different TKs including VEGFR1, VEGFR2, VEGFR3 and other growth factors (Mendel et al. 2003). In contrast to sunitinib, 22SYM is recently developed TK inhibitor $\left(\mathrm{IC}_{50}=15.3 \mathrm{nM}\right.$, VEGFR2 TK) derived from oxazolamine compound AAZ that inhibits selectively VEGFR2 TK (Lintnerová et al. 2014).

Our results demonstrating inhibition of ERK1/2 and Akt phosphorylation are in line with anti-angiogenic activity of 22SYM based on inhibited migratory capabilities of bovine endothelial cells in the wound healing assay and in vivo angiogenesis in the Zebrafish embryo assay (Lintnerová et al. 2014). In summary, the present study showed that 22SYM inhibits proliferation and migration of HUVECs stimulated by VEGF-A, and proved that 22SYM inhibits phosphorylation of Akt and ERK1/2. Our results suggest that 22SYM possesses potent anti-angiogenic activity through inhibition of the Ras/Raf/MEK/ERK1/2 and PI3K/Akt dependent signal pathways. Lower inhibition of 22SYM in comparison with sunitinib in the wound healing HUVECs assay can reflect its higher specific action as well as a lower bioavailability of 22SYM because it was optimized for sunitinib before its clinical registration. However, specific binding of 22SYM to VEGFR2 TK may decrease potential side effects and toxicity in comparison with multi kinase inhibitors.

Acknowledgement. This study was supported by grants APVV0291-12, VEGA 1/0557/15 and VEGA 1/0634/13. Authors thank to Lucia Lintnerová for the synthesis of 22SYM. We are grateful to Pfizer Inc. for a gift of sunitinib L-malate.

\section{References}

Cantley L. C. (2002): The phosphoinositide 3-kinase pathway. Science 296, 1655-1657

http://dx.doi.org/10.1126/science.296.5573.1655

Carmeliet P., Jain R. K. (2000): Angiogenesis in cancer and other diseases. Nature 407, 249-257

http://dx.doi.org/10.1038/35025220

Faivre S., Demetri G., Sargent W., Raymond E. (2007): Molecular basis for sunitinib efficacy and future clinical development. Nat. Rev. Drug. Discov. 6, 734-745 http://dx.doi.org/10.1038/nrd2380

Ferrara N. (2010): Pathways mediating VEGF-independent tumor angiogenesis. Cytokine Growth Factor Rev. 21, 21-26 http://dx.doi.org/10.1016/j.cytogfr.2009.11.003

Folkman J. (2007): Angiogenesis: an organizing principle for drug discovery? Nat. Rev. Drug Discov. 6, 273-286 http://dx.doi.org/10.1038/nrd2115

Ivy S. P., Wick J. Y., Kaufman B. M. (2009): An overview of smallmolecule inhibitors of VEGFR signaling. Nat. Rev. Clin. Oncol. 6, 569-579 http://dx.doi.org/10.1038/nrclinonc.2009.130

Lintnerová L., García-Caballero M., Gregáň F., Melicherčík M., Quesada A. R., Dobiaš J., Lác J., Sališová M., Boháč A. (2014): A development of chimeric VEGFR2 TK inhibitor based on two ligand conformers from PDB: 1Y6A complex - medicinal chemistry consequences of a TKs analysis. Eur. J. Med. Chem. 72, 146-159 http://dx.doi.org/10.1016/j.ejmech.2013.11.023

Mendel D. B., Laird A. D., Xin X., Louie S. G., Christensen J. G., Li G., Schreck R. E., Abrams T. J., Ngai T. J., Lee L. B. et al. (2003): In vivo antitumor activity of SU11248, a novel tyrosine kinase inhibitor targeting vascular endothelial growth factor and platelet-derived growth factor receptors: determination of a pharmacokinetic/pharmacodynamic relationship. Clin. Cancer Res. 9, 327-337

Zachary I. (2001): Signaling mechanisms mediating vascular protective actions of vascular endothelial growth factor. Am. J. Physiol. 280, C1375-1386

Received: September 9, 2015

Final version accepted: December 17, 2015

First published online: July 22, 2016 